\title{
Bio-inspired 3D-printed piezoelectric device for acoustic frequency selection
}

\author{
Roger Domingo-Roca ${ }^{a *}$, Benjamin Tillera, Joseph Curt Jackson ${ }^{a}$, James Frederick Charles Windmilla
}

Department of Electronic \& Electrical Engineering, University of Strathclyde, Glasgow, United Kingdom

*Corresponding author. E-mail: roger.domingo-roca@strath.ac.uk (R. Domingo-Roca)

a Centre for Ultrasonic Engineering, Dept. Electronic \& Electrical Engineering, 204 George Street, University of Strathclyde, Glasgow, G1 1XW, United Kingdom

Abstract | Development of 3D-printed devices, sensors, and actuators has become increasingly popular in recent years due to low cost, rapid production, and device personalization. This personalization process allows the development of devices with unique physical properties and phenomena that enhance the desired properties of the 3D-printed part. Biomimetics is a technique used to develop engineered devices, as organisms present in nature can provide smart and simple solutions to complex problems across a wide range of applications. Locust ears have a simple tympanic membrane with varying thicknesses that allows acoustic frequency selection, as well as presenting nonlinear phenomena. This acoustic frequency selection assists the insect in predation and swarming. This work presents the development of a piezoelectric polymeric material that has been used to 3D-print a new frequency selective piezoelectric sensor inspired by the locust's tympanic membrane. Such 3D-printing of functional sensors and actuators provides an insight into the development and enhancement of polymer-based science, with exciting and promising potential for the near future.

KEYWORDS: Locust; Biomimesis; Piezoelectric; Polymer; 3D-printing

\section{Introduction}

Biological systems display simple and yet efficient adaptations to accomplish specific functions and address complex challenges. Observation and understanding of these adaptations can provide inspirations for solutions that can be applied to solve modern human problems. This approach, known as bio-inspiration, has become a very common tool during recent years, turning into a rapidly-growing field of research with applications in medicine [1], chemistry [2,3], materials science [4,5], robotics [6,7], and more. One approach can be found in the study of insect acoustic systems inspiring the design of novel artificial acoustic sensors, as well as developing signal processing systems and hearing aid devices $[8,9]$. Contingent on the species, insects present different ways of detecting and processing sound. This includes some that have tympanal ears consisting of a membrane stretched over a fluid-filled cavity, onto which neural sensors (scolopidia) attach. When the membrane moves due to incoming sounds, the mechanical motion is transduced by the scolopidia into an electrical signal that is interpreted by the central nervous system [10]. One of the many insects presenting this kind of acoustic system is the locust, whose tympanal membrane (TM) consists of a pearshaped membrane of cuticle with variable thickness that selectively responds to specific frequencies at different regions of the membrane [11,12] (Fig. 1a), and which has been widely studied since the discovery of travelling waves (TWs) spreading across it [13].

3D-printing technology has become a growing research field because of its wide range of applications such as biomedical engineering $[14,15]$, mechanics $[16,17]$, electronics $[18,19]$, and aerospace $[20,21]$ among others. The development of micro-devices requires small and complex structures that can be built rapidly and at low cost yet, typically, the most common polymer-based microelectromechanical systems (MEMS) approaches are slow and time consuming. 3D printing techniques, whether they use solid-, liquid-, or powder-based approaches, permit the rapid production of complex and accurate geometries from computer-aided design (CAD) files, with polymers as the materials that play the most prominent roles amongst commercial 3D-printable materials. One of the main disadvantages of these materials when using them for rapid prototyping purposes is that the functionality of the resulting parts solely relies on their mechanical and geometric properties. However, providing new functionalities to these materials becomes relatively easy when embedding specific ceramics and/or metals within the polymeric matrix. Liquidbased approaches using light-responsive polymers facilitate the development of functional 3D-printable composites as they enable a quick and simple suspension of nanostructures within the photo-curable polymeric matrix, which solidifies after exposure to ultraviolet (UV) light.

When developing a photocurable 3D-printable material there are four main considerations to take into account; (i) the oligomers that will compose the polymeric structure, (ii) the species that will start the photopolymerization process (photoinitiator), (iii) the UV blocker that will avoid light scattering and over-curing during the 3D-printing process, and (iv) the nanostructures used as fillers. Thus, to ensure a good 3D-printing resolution, SUDAN I was used as a dye as it can be easily dissolved within the liquid polymer matrix. SUDAN I's azobenzene moieties are light-sensitive, blocking the UV light and thus avoiding Mie scattering $\left(d_{N P} \approx \lambda_{u v}\right)$, therefore improving 3D-printing resolution. Moreover, accurately controlling the amount and type of dye, it is possible to finely tune the mechanical properties of the resulting composite due to the azobenzene moieties' light-responsive properties [22], arising from the $\pi$-electrons in the p-orbitals of the $\mathrm{sp}^{2}$ hybridized nitrogen atoms. 

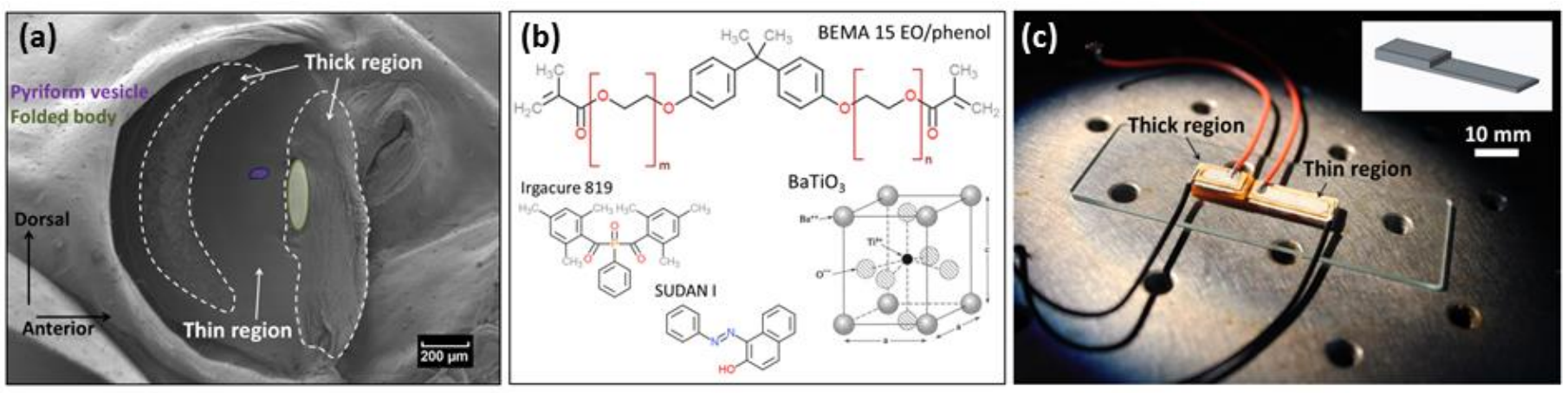

Fig. 1: (a) SEM image of the locust TM. Thick regions are delimited within dashed lines, and the PV and the FB are also highlighted. Image first published in Tympanal travelling waves in migratory locusts [13]. (b) Monomer, photoinitiator, dye, and NPs crystallographic structure [38]. (c) Locust TM bio-inspired 3D-printed sensor and its corresponding CAD file. The standardized dimensions of the 3D-printed device are shown in Fig. S3 of the ESIT.

$\mathrm{BaTiO}_{3}$ is a widely-studied piezoelectric ceramic material $[23,24]$, both in its bulk form and its different nanosctructures, being paraelectric (cubic) above $125^{\circ} \mathrm{C}$, and ferroelectric (tetragonal) between $8-25^{\circ} \mathrm{C}$, which makes it suitable for several applications [24]. Using $\mathrm{BaTiO}_{3}$ dots, rods, or nanotubes highly influences its ferroelectric properties due to the imbalance between the Coulomb interactions (long-range interactions, modified due to a lack of periodicity) and the covalent interactions (short-range interactions, modified near the surface boundary) caused by atomic off-centre displacements, reaching $d_{33}$ values above $400 \mathrm{pC} / \mathrm{N}$ when using specific arrangements [25]. Though it is believed that ferroelectricity would completely disappear in nanostructures below a critical size [26], the ferroelectric properties of $\mathrm{BaTiO}_{3}$ NPs have reported that larger NP size present higher $d_{33}$ values [27]. When embedding ceramic piezoelectric NPs within a polymeric matrix, the piezoelectric domains stay randomly oriented within the polymer chains after the photopolymerization process, making the application of an electric field $(\boldsymbol{E})$ to force the electric charges to redistribute themselves microscopically necessary, resulting in a macroscopic polarization of the sample that depends on the anisotropy of the material. If the applied $\boldsymbol{E}$ is not large enough, the electric charges will not receive enough energy to be reoriented in the direction of the field, whereas if it is too high, it leads to electric breakdown of the sample due to the excess of provided energy. Different ceramic piezoelectric materials, which are transversely isotropic, have distinct ranges of optimum poling $\boldsymbol{E}$, as its internal energy density must remain positive since it must be minimal in a state of equilibrium [28]. Nevertheless, when dealing with composites that include piezoelectric NPs, factors like NPs concentration, size, and distribution play an important role [29]. Temperature, $T$, is also a key parameter to control during the poling process as the application of $\boldsymbol{E}$ above the Curie temperature, $T_{c}$, and during a controlled decrease of $T$ would be more efficient as the process is energetically favourable. Nevertheless, in most cases the melting temperature of the polymer is below $T_{c}$. Furthermore, before 3D-printing, the optimum NP size must be selected to ensure a proper balance between 3D-printing resolution and piezoelectric output.

In this work we use the simple, but smart, structure of the locust's TM as inspiration to develop a new piezoelectric sensor able to discern between different acoustic frequencies. This utilised a 3D-printable UV-light responsive piezoelectric composite built by stereolithography (STL), consisting of barium titanate $\left(\mathrm{BaTiO}_{3}\right)$ nanoparticles (NPs) embedded within a polymerisable methacrylate group linked to bisphenol by ethylene oxide (EO) chains of different length (Fig. 1b).

\section{Theoretical and Experimental Methods}

Two different 3D-printable polymers were synthesized to perform this work using bisphenol-A ethoxylate dimethacrylate (BEMA), phenylbis (2,4,6-trimethylbenzoyl) phosphine oxide (Irgacure 819), SUDAN I, silver paint (all of them obtained from Sigma-Aldrich), and $\mathrm{BaTiO}_{3} 500 \mathrm{~nm}$ nanoparticles (NPs) purchased from US Research Nanomaterials, Inc. (Houston, TX, USA).

\subsection{Synthesis of BEMA}

3D-printable BEMA was developed by mixing BEMA with Irgacure 819 at $1 \mathrm{wt} \%$. The mixture was left under magnetic stirring for 24 hours to ensure cross-linking of the polymer chains. 0.2 wt\% of SUDAN I was then added with respect to BEMA and the whole mixture was put into a THINKY AER-250 mechanical mixer, mixing the composite for 3 minutes at 1500 rpm and de-foaming it for 2 minutes at $1200 \mathrm{rpm}$. Finally, the mixture was sonicated for 5 minutes before 3D-printing. A control sample was made using a screen-printing technique with layer thickness of $50 \mu \mathrm{m}$, using an R\&K control coater, curing every layer under UV light for 1 minute using an Intertronics IUV250 Hand Lamp (Intertronics, Kidlington, England, UK).

\subsection{Synthesis of $\mathrm{BaTiO}_{3} @ B E M A$}

To develop the piezoelectric polymer-based material, the process described in sub-section 2.1 was followed. In this case, though, a $0.1 \mathrm{wt} \%$ of SUDAN I was used with respect to BEMA as a $0.2 \mathrm{wt} \%$ loading blocked the majority of the UV light, resulting in a failure to 3D-print a device. In addition, $\mathrm{BaTiO}_{3}$ NPs were added into the mixture at a 33 wt\%. Such a percentage was chosen because lower wt\% of NPs gave no piezoelectric measurable signal, and higher wt\% of NPs resulted in poor 3D-printing quality. A control sample was built following the same process described in sub-section 2.1.

Both mixtures were used with an ASIGA PicoPlus27 digital light processing stereolithography (DLP-SLA) 3D printer (ASIGA, Anaheim Hills, California, USA), and 3D-printed at $10 \mu \mathrm{m}$ slice thickness, adjusting the 3D-printing parameters in order to give a reasonable balance between printing time and printing accuracy. 


\subsection{D-printing of devices}

Firstly, a support structure was 3D-printed on which the sensors lies, using a commercial material provided by ASIGA with robust mechanical properties, consisting of a rectangle 30 $\mathrm{mm}$ long, $7 \mathrm{~mm}$ wide, $1 \mathrm{~mm}$ height, and a wall thickness of 1 $\mathrm{mm}$. By stopping the 3D-printing process at a specific build layer it was possible to swap the build fluid to 3D-print layers of BEMA, which was used to 3D-print the base of the membrane $(0.66 \mathrm{~mm})$. When the desired thickness was reached, the build fluid was swapped to $\mathrm{BaTiO}_{3} @ \mathrm{BEMA}$, which was used to 3D-print a piezoelectric layer of $0.5 \mathrm{~mm}$ onto the thin membrane. When the process was finished, the build fluid was changed back to BEMA, which was used to 3D-print the main part of the thick membrane of the sensor $(1.67 \mathrm{~mm})$, and it was finally changed again to $\mathrm{BaTiO}_{3} @ \mathrm{BEMA}$ to 3D-print the last piezoelectric layer $(0.5 \mathrm{~mm})$. During this process, silver paint electrodes were carefully applied between the piezoelectric layers in order to attach electrical connections.

Flat samples of BEMA loaded with different wt\% of $\mathrm{BaTiO}_{3} \mathrm{NPs}$ were also prepared by STL to perform nanoindentation assays in order to investigate their effect on the mechanical properties of the photo-curable polymeric formulations.

\subsection{Mechanical properties}

Mechanical properties of the developed material were evaluated using an MFT 3D Nanoindenter and the data was treated with IBIS software.

Load-displacement, $P$ - $h$, load-unload curves were obtained using a calibrated Berkovich tip made of single crystalline diamond. The penetration depth was kept below $10 \%$ of the sample's thickness in order to prevent the substrate's influence on the mechanical properties [30]. The values of the reduced elastic modulus $\left(E_{r}\right)$ and hardness $(H)$ were determined using the method of Oliver and Pharr [31]. The obtained data was treated in order to correct for thermal drift, the instrument compliance, the indenter shape function, and the initial penetration depth. Flat samples of BEMA loaded with different wt\% of $\mathrm{BaTiO}_{3} \mathrm{NPs}$ were measured in arrays of 6x6 indents, separated from each other by $15 \mu \mathrm{m}$, and covering a total area of $90 \mu \mathrm{m}^{2}$. This separation between indents was imposed to avoid the influence of neighbour indentations. The maximum applied load was $100 \mu \mathrm{N}$.

\subsubsection{The Sato and Furukawa model}

When adding filler within a polymer matrix, the adhesion between the two components plays a key role in the resulting mechanical properties of the composite. Sato and Furukawa [32] developed a mathematical model to predict the behaviour of the composite as a function of the adhesion parameter, $j$, which takes a value of 1 for poor adhesion, and a value of 0 for perfect adhesion (Eq. 1, and Eq. 2). In the case of no adhesion between the components, the filler cannot carry any load, so all the load must be carried by the polymer. On the other hand, in the case of perfect adhesion, shear effects around the filler, stresses in the polymer, and effects due to the Poisson's ratio can be neglected in this analysis. From these assumptions, Sato and Furukawa developed the expressions:

$$
\begin{gathered}
E_{c}=E_{m}\left[\left(1+\frac{\varphi_{f}^{2 / 3}}{2-2 \varphi_{f}{ }^{1 / 3}}\right)(1-j \psi)-\frac{\varphi_{f}^{2 / 3} j \psi}{\left(1-\varphi_{f}^{1 / 3}\right) \varphi_{f}}\right] \\
\psi=\left(\frac{\varphi_{f}}{3}\right) \frac{1+\varphi_{f}^{1 / 3}-\varphi_{f}^{2 / 3}}{1-\varphi_{f}^{1 / 3}+\varphi_{f}^{2 / 3}}
\end{gathered}
$$

where $E_{c}$, and $E_{m}$ are the elastic modulus of the composite and the matrix, respectively, and $\varphi_{f}$ is the filler volume fraction.

\subsection{Acoustic response}

The acoustic response of the 3D-printed device was assessed using a 3D laser Doppler vibrometer (3D LDV) system with an MSA-100-3D scanning head (Polytec, Waldbrom, Germany). The acoustic response of the 3D-printed device was measured under acoustic wideband stimulation ( 0.3 to $17 \mathrm{kHz}$ using a 4" 40W loudspeaker, Maplin Electronics Ltd., Barnsley, England, in the far-field regime) by simultaneously recording the velocity of motion of the sensor and the sound pressure level (SPL dB) near it using a Bruel \& Kjaer (B\&K) 4138 precision pressure microphone (Naerum, Denmark) and preamplifier (Bruer \& Kjaer, 2633), with its diaphragm parallel to the sound direction to maximize the response and using a calibrated stimulus sound level of $65 \mathrm{~dB}$ re $20 \mu \mathrm{Pa}$ ( $35 \mathrm{mPa}$ ), as schematically shown in Fig. 2.

\subsubsection{Travelling wave analysis}

The propagation of the TWs was studied using the 3D LDV by drawing a transect line from edge to edge of the membrane for five different frequencies. The motion across the membrane becomes apparent when the response is displayed for every $10^{\circ}$ of phase. TWs can be characterized as a part of the analysis of the vibration of the 3D-printed sensor, as they move in time, making the vibration change with both position and time, and their velocity and wavelength can be determined using Eq. 4, and Eq. 5 [13].

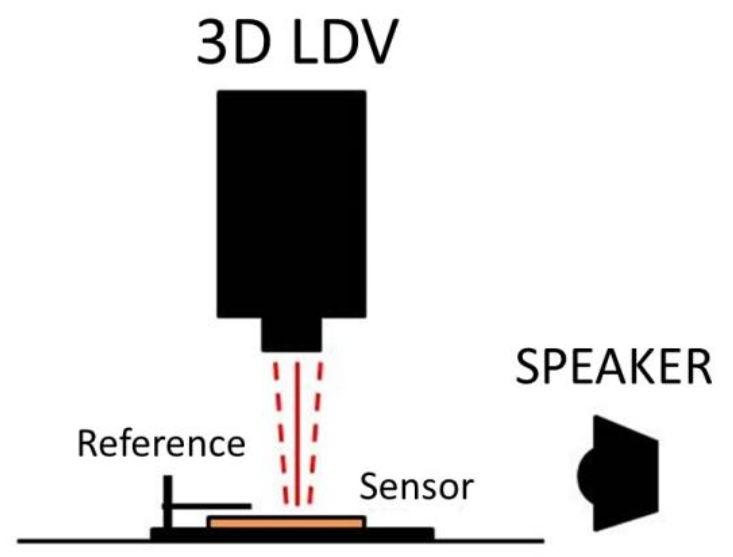

Fig. 2: Schematic set up to test the acoustic response of the 3D-printed sensor. The loudspeaker sits behind the sensor at a distance that ensures the far-field regime, and the 3D-LDV head stays on top of the sensor. The sensor was fixed on the table, and a Bruer\&Kjaer (B\&K) 4138 microphone was used as a reference.

$$
\begin{gathered}
\delta_{t}=\delta_{\phi} / 2 \pi f \\
V_{W}=\delta_{x} / \delta_{t}
\end{gathered}
$$




$$
L_{W}=2 \pi \delta_{x} / \delta_{\phi}
$$

where $f$ is the frequency, $\delta_{\phi}$ is the phase difference between the two points, $\delta_{t}$ is the travel time, and $\delta_{x}$ is the distance travelled. $V_{W}$ and $L_{W}$ are the velocity and the wavelength of the TW, respectively.

\subsection{Piezoelectric analysis}

Both the direct and converse piezoelectric responses were studied (Fig. 4, and Fig. 5) after poling the device at an electric field of 2.4 $\mathrm{kV} / \mathrm{mm}$ for one hour. The output voltage was measured when the sensor was excited at single frequencies within the range of $1 \mathrm{kHz}$ to $17 \mathrm{kHz}$, recording both the reference (Fig. 4a), and the piezoelectric response (Fig. 4b) at all frequencies. Bursts of 2 cycles every $100 \mathrm{~ms}$, at an amplitude of $20 \mathrm{~V}_{\mathrm{pp}}$ were used, varying the distance between the loudspeaker and the sensor from $35 \mathrm{~cm}$ to 10 $\mathrm{cm}$ in order to properly discern between the electromagnetic and the acoustic response. An acoustic amplifier was needed to reach high SPL dB values in order to get an electrical output from the sensor due to its low piezoelectric coefficient, measured to be $d_{33} \sim$ $3 \mathrm{pC} / \mathrm{N}$ using the 3D LDV [33,34]. Moreover, a charge amplifier (factor 475) was also needed to properly record an electrical signal caused by direct piezoelectric effect of the 3D-printed device. The voltage difference, $\Delta V$, was normalized against the $B \& K$ microphone output.

\section{Results and Discussion}

\subsection{Mechanical properties}

The values of $E_{r}$ and $H$ determined from the $P$-h load-unload curves (Fig. 3a) using the method of Oliver and Pharr are shown in Table I. As nanoindentation provides $E_{r}$ instead of $E$, the material's Poisson's ratio was assumed to be 0.4 .

Table I: Values of Young's modulus and hardness values obtained from the P-h curves using the method of Oliver and Pharr.

\begin{tabular}{lcc}
\hline \multicolumn{1}{c}{ Sample } & $\begin{array}{c}\text { Young's modulus } \\
{[\mathrm{Mpa}]}\end{array}$ & $\begin{array}{c}\text { Hardness } \\
{[\mathrm{Mpa}]}\end{array}$ \\
\hline BEMA & $3.070 \pm 0.010$ & $0.7442 \pm 0.0054$ \\
BEMA:BTO(33) & $3.049 \pm 0.035$ & $0.7130 \pm 0.0028$ \\
BEMA:BTO(50) & $2.955 \pm 0.040$ & $0.725 \pm 0.011$ \\
BEMA:BTO(75) & $5.670 \pm 0.052$ & $1.610 \pm 0.010$
\end{tabular}

Although smooth changes of the mechanical properties are observed when increasing the $\mathrm{BaTiO}_{3}$ wt\%, attributed to NPs agglomeration (Fig. 3b), significant changes on both $E$ and $H$ are not observed up to loadings higher than $50 \mathrm{wt} \%$, following the Sato and Furukawa model [32], which suggests good adhesion between the filler and the matrix. NPs agglomeration can be prevented using surfactants [35], and some studies have revealed that viscous media also help its prevention $[35,36]$ when using polymers with long EO chains, producing a decrease of the existing Van der Waals forces between NPs. One of the advantages of BEMA with respect to other 3D-printable polymeric matrices and other commercial materials is the presence of long EO chains, which leads to a decrease of NPs aggregation due to an increase of viscosity of the fluid.
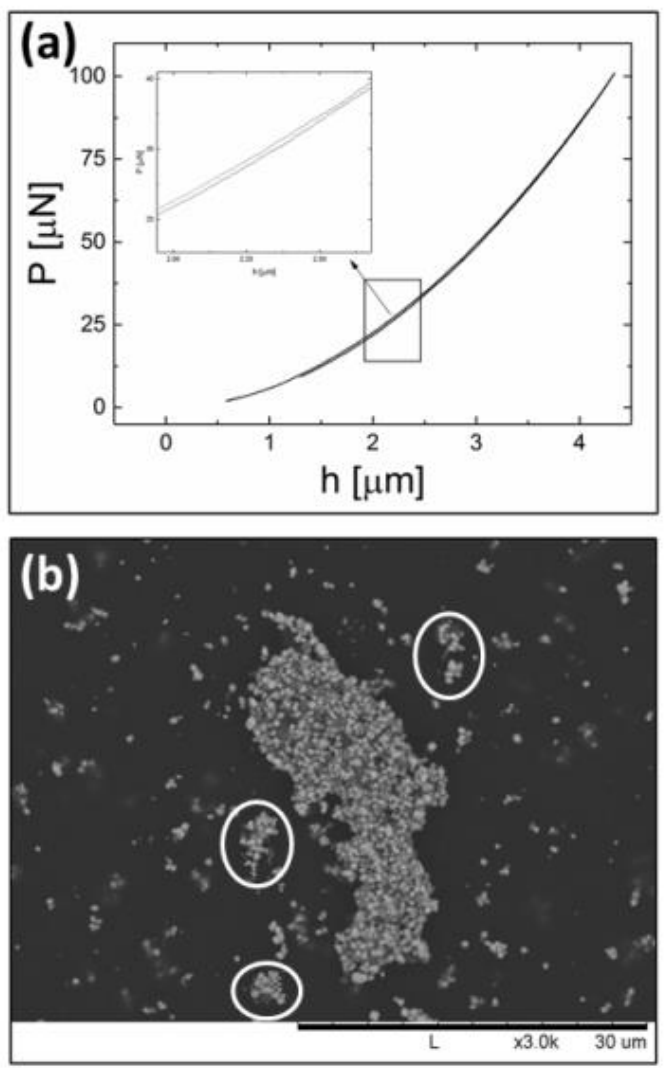

Fig. 3: (a) shows the $P$ - $h$ curve for BEMA, applying a maximum load of 100 $\mu \mathrm{N}$ and reaching a maximum penetration depth of $4.34 \mu \mathrm{m}$. All the samples presented the same elastic behaviour, recovering its initial position when the load was removed. A plastic component in the $P$ - $h$ curves is observed when measuring samples with high weight loadings. (b) SEM image of a

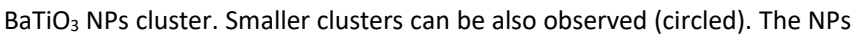
average diameter is $500 \mathrm{~nm}$, as provided by the manufacturer (US Research Nanomaterials, Inc.).

\subsection{Acoustic response and TW analysis}

More than 3000 points were measured, providing an accurate representation of the membrane's motion via a contactless process, and allowing the measurement of the average displacement of the different regions of the sensor at specific frequencies using the fast Fourier transform (FFT) module of the 3D LDV system, which were normalized with the SPL dB (Table II). The first 6 resonant frequencies of the device were measured to be $0.86,2.44,3.55,4.68,5.41$, and $8.12 \mathrm{kHz}$.
Table II: Values of the average amplitude of motion of the thick and thin regions of the 3D-printed piezoelectric sensor in the vertical direction ( $z$ axis) under single frequency acoustic stimulation. 


\begin{tabular}{ccccc}
$\begin{array}{c}\text { Frequency } \\
{[\mathrm{kHz}]}\end{array}$ & $\begin{array}{c}\boldsymbol{z} / \mathrm{dB} \\
{[\mathrm{pm} / \mathrm{dB}]}\end{array}$ & $\begin{array}{c}z / \mathrm{Pa} \\
{[\mathrm{nm} / \mathrm{Pa}]}\end{array}$ & $\begin{array}{c}z / \mathrm{dB} \\
{[\mathbf{p m} / \mathbf{d B}]}\end{array}$ & $\begin{array}{c}z / \mathrm{Pa} \\
{[\mathrm{nm} / \mathrm{Pa}]}\end{array}$ \\
\hline 0.60 & 11.80 & 53.23 & 9.62 & 44.18 \\
0.80 & 15.16 & 29.40 & 9.82 & 19.04 \\
1.00 & 5.79 & 12.34 & 2.91 & 5.085 \\
1.90 & 14.60 & 13.39 & 44.53 & 51.79 \\
2.20 & 8.35 & 7.51 & 39.24 & 65.081 \\
3.00 & 11.63 & 22.19 & 14.58 & 30.97 \\
5.00 & 7.41 & 6.67 & 2.80 & 5.51 \\
8.00 & 4.52 & 2.24 & 6.38 & 9.27 \\
10.00 & 2.73 & 3.80 & 1.78 & 7.38 \\
\hline
\end{tabular}

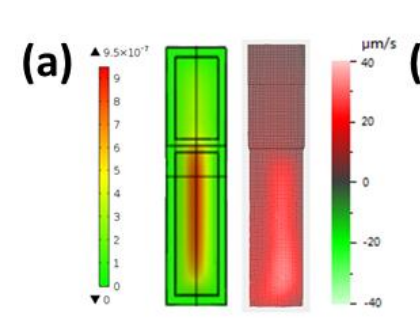

(b)
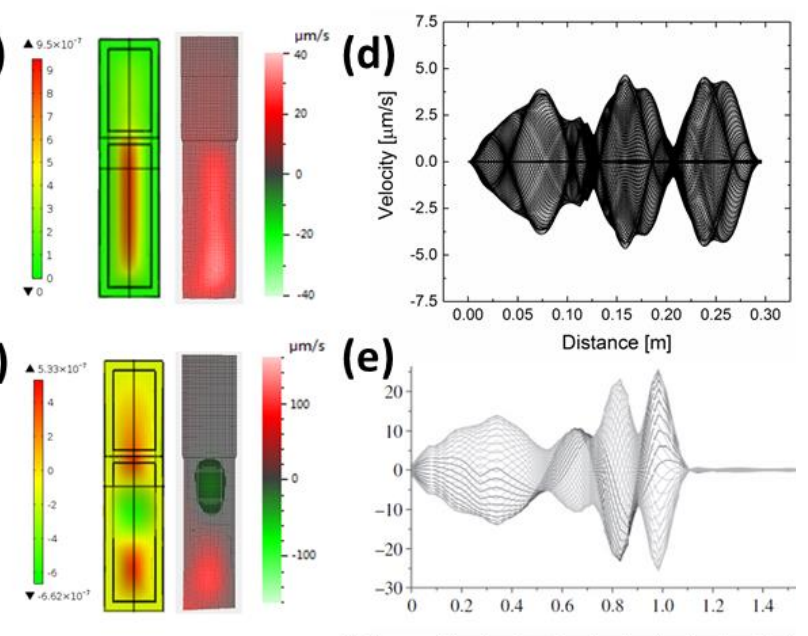

(e)
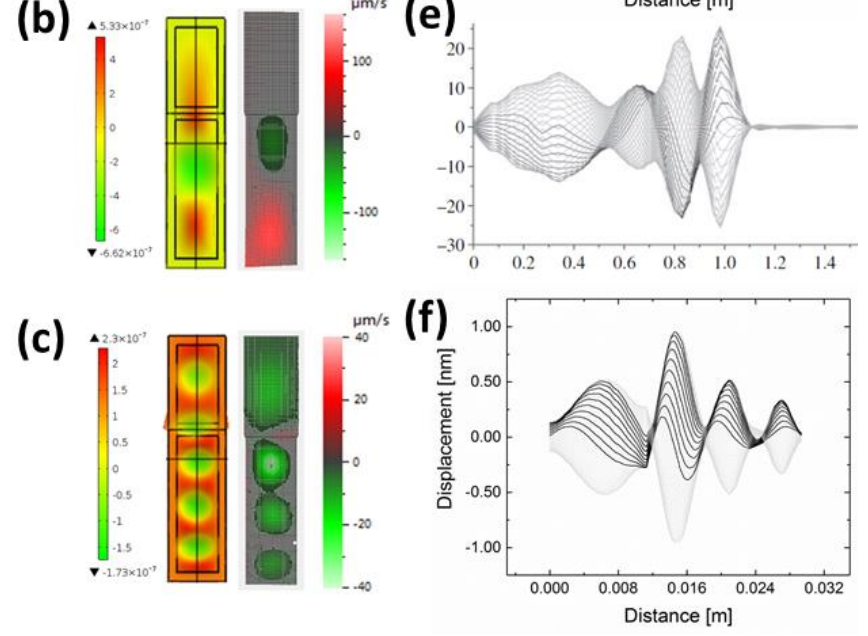

Fig. 4: (a-c) Simulation and experimental mechanical deflection of the 3Dprinted sensor at $1 \mathrm{kHz}, 2.2 \mathrm{kHz}$, and $5 \mathrm{kHz}$ respectively, and their corresponding scales. (d-f) Show the TWs observed in $\mathrm{COMSOL}^{\circledR}$, the locust $\mathrm{TM}$, and the 3D-printed sensor, respectively.

When acoustically stimulated by single-frequency inputs, the 3D-printed device displayed larger amplitudes of motion at frequencies below the first resonant frequency on the thick region, whereas the opposite trend was observed at higher frequencies on the thin region (Table II). Simulation techniques using $\mathrm{COMSOL}^{\circledR}$ confirmed the experimental behaviour (Fig. 3a,b,c). Furthermore, the presence of travelling waves (TW) was observed by 3D LDV analysis and also confirmed by $\mathrm{COMSOL}^{\circledR}$ simulation techniques (Fig. $3 \mathrm{~d}$ ), revealing an increase of the TW's velocity of propagation $\left(v_{T W}\right)$ with frequency on the thin membrane at low frequencies, and a decrease of $v_{T W}$ with frequency on the thick membrane at high frequencies (Table III), giving values close to the material's speed of sound, which is determined by Eq. 6 .

$$
c=\sqrt{B / \rho}
$$

where $B$ is the bulk modulus of the material, and $\rho$ is its density. Thus, from the results obtained from the nanoindentation assays the speed of sound in BEMA is 52.57 $\mathrm{m} / \mathrm{s}$, decreasing to $49.62 \mathrm{~m} / \mathrm{s}$ when BEMA is loaded with $\mathrm{BaTiO}_{3} \mathrm{NPs}$ at a $33 \mathrm{wt} \%$. For each frequency, the deflections of the membrane do not stay in the same position, but they travel across the membrane irrespective of the incidence angle of the sound field, modifying its envelope shape when varying the driving frequency.

Table III: Values of the velocities and wavelengths of the TWs measured onto the 3D-printed sensor.

\begin{tabular}{ccccc}
\hline Frequency & \multicolumn{2}{c}{ Thick region } & \multicolumn{2}{c}{ Thin region } \\
{$[\mathrm{Hz}]$} & $\boldsymbol{v}[\mathrm{m} / \mathrm{s}]$ & $\boldsymbol{\lambda}[\mathrm{mm}]$ & $\boldsymbol{v}[\mathrm{m} / \mathrm{s}]$ & $\boldsymbol{\lambda}[\mathrm{mm}]$ \\
\hline 2.2 & - & - & 27.79 & 11.21 \\
3.0 & - & - & 51.52 & 17.17 \\
5.0 & 71.63 & 14.33 & 30.48 & 5.77 \\
8.0 & 53.92 & 6.74 & - & - \\
9.0 & 48.29 & 5.37 & - & - \\
\hline
\end{tabular}

These behaviours match those observed on the locust's TM [13] (Fig. 3e), suggesting that the presence of TWs does not depend of any biological active process, but it is a phenomenon highly reliant on the geometry and structure of the membrane, in effect due to nonlinearities of the system. When a single-frequency sound stimulus impinges the ear, the local pressure changes periodically with time and the deformation of the membrane can be described by a nonlinear wave equation. There is a characteristic place in the membrane where the frequency of the wave is matched by the frequency of the critical oscillators that describe the system. Far from this resonance point, the wave equation describes traveling waves that are linear for small vibration amplitudes. The fall in wave velocity implies an increase in the amplitude of the wave, leading to an increase of displacement of the wave as it approaches the resonant point [37]. Nevertheless, the direction of propagation of the TWs has been observed to be opposite to that measured on the locust TM for frequencies higher than $1 \mathrm{kHz}$, as confirmed by $\mathrm{COMSOL}^{\circledR}$ simulation. The mechanoreceptors attachment points (folded body, FB, and pyriform vesicle, PV, Fig. 1a) on the locust TM could explain this difference between the biological system and the 3D-printed sensor, as they contribute a local increase of mass at specific regions of the locust TM. Furthermore, a non-perfect attachment of the 3D-printed membrane with the support structure might also play an important role on the direction of propagation of the TWs, as well as contributing to a non-perfect experimental modal shape (Fig. 3a, 3b, 3c). Notwithstanding, a phenomenological match of the acoustic response was observed between the 3D-printed sensor and the locust TM.

\subsection{Piezoelectric analysis}

The piezoelectric response of the sensor to both the converse and direct piezoelectric stimulation is shown in Fig. 5 and Fig. 6, respectively. 


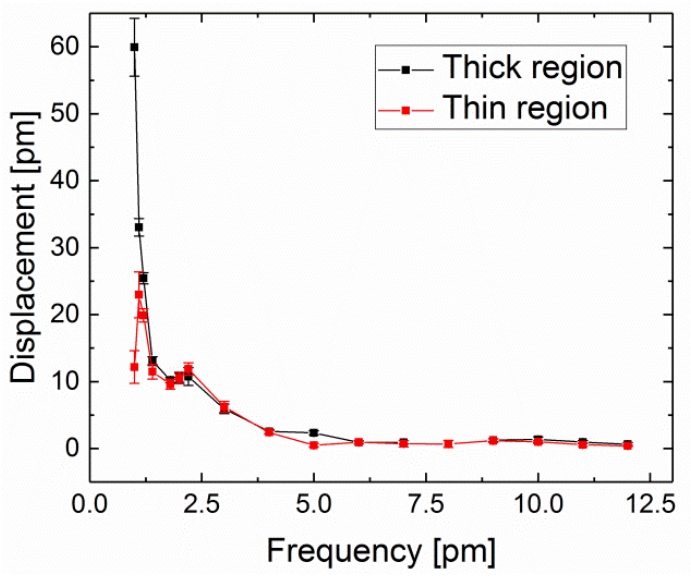

Fig. 5: Average displacement values of the different regions of the 3Dprinted bio-inspired piezoelectric sensor when electrically stimulated within the frequency range of $1 \mathrm{kHz}$ to $17 \mathrm{kHz}$ with the corresponding error bars.
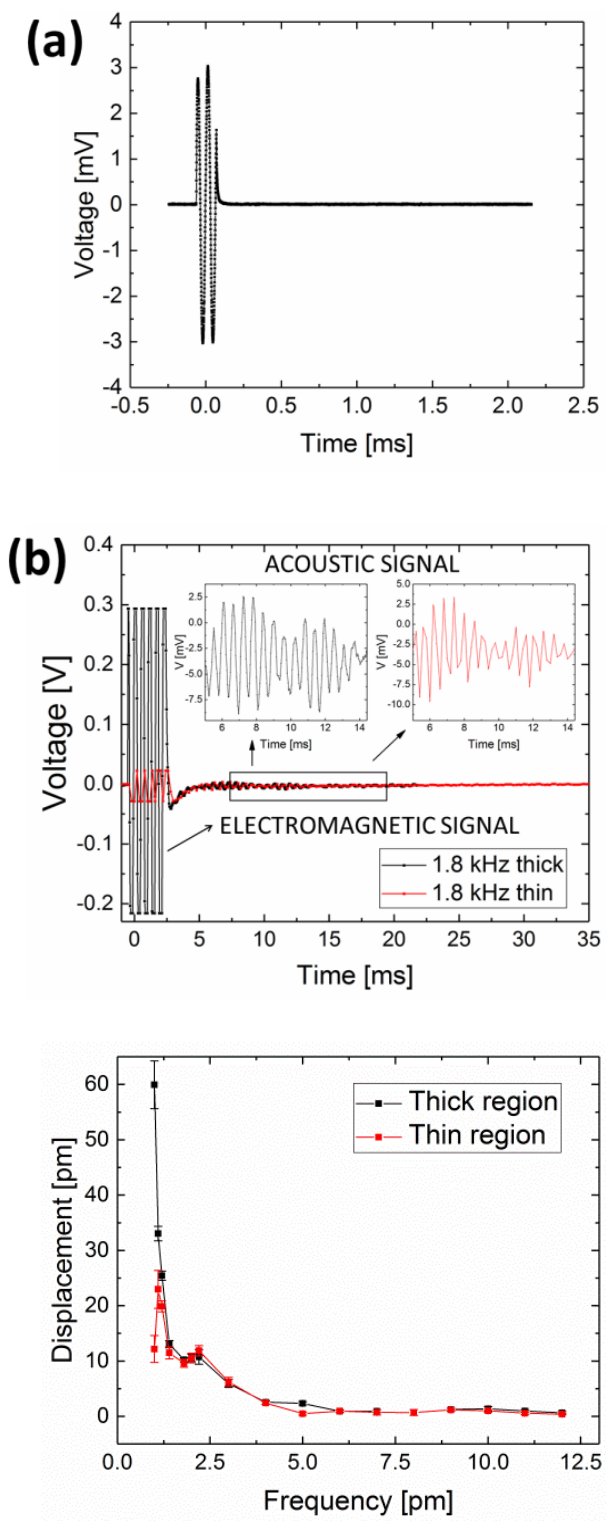

Fig. 6: (a) Shows the acoustic input signal at $15 \mathrm{kHz}$ consisting of a burst of 2 cycles every $100 \mathrm{~ms}$. (b) Shows the response of the 3D-printed device with its two well-differentiated regions at $1.8 \mathrm{kHz}$. The response is divided between the electromagnetic and the acoustic responses. The same experiment was performed within the range of $1 \mathrm{kHz}$ to $17 \mathrm{kHz}$ in steps of 1 $\mathrm{kHz}$. (c) Shows the normalized values of the output voltage of the different regions under acoustic stimulation within the same frequency range, revealing larger output voltages in the thick region at all the studied frequencies.
Even though the sensor seemed to respond to all the frequencies within the range of $1 \mathrm{kHz}$ to $17 \mathrm{kHz}$, it was only possible to clearly discern an electrical output arising from acoustic signals at $1.8 \mathrm{kHz}$, $2.2 \mathrm{kHz}, 9 \mathrm{kHz}$, and $10 \mathrm{kHz}$, as shown in Fig. 6c. The two regions of the 3D-printed sensor were poled at an electric field of $2.4 \mathrm{kV} / \mathrm{mm}$, for $1 \mathrm{~h}$ at $120^{\circ} \mathrm{C}$ in silicon oil.

Due to all the parameters that must be taken into account when developing a piezoelectric 3D-printable material, it was not trivial to obtain a clear piezoelectric signal at all the frequencies. Therefore, the same experiments using the same set up were performed using PVDF films instead of the piezoelectric 3Dprintable material, leading to the same acoustic frequency selection observed on the 3D LDV under acoustic stimulation (ESIt, PVDF data). Thus, to confirm the piezoelectric behaviour of the 3Dprintable piezoelectric material, the converse piezoelectric effect was tested for the two different regions of the sensor using sinusoidal inputs at different frequencies at amplitude of $10 \mathrm{~V}$, and the mechanical deflection was measured using the 3D LDV technique (Fig. 5). Mechanical deflection of the sensor was successfully measured on both the thick and thin regions of the 3Dprinted device. Reliable results were obtained up to $5 \mathrm{kHz}$, where higher amplitudes of motion were obtained below $3 \mathrm{kHz}$, and mechanical deflections of the same order of magnitude were obtained from $3 \mathrm{kHz}$ to $5 \mathrm{kHz}$. At higher frequencies, all the values were measured to be at noise level. Nevertheless, when the $d_{33}$ coefficient of the 3D-printable piezoelectric material is improved, the response of the sensor is expected to be like the one shown in Fig. S5 (ESIt, PVDF data), where clear acoustic frequency selectivity can be observed.

\section{Conclusions}

A 3D-printable piezoelectric material suitable for 3D-printing techniques using the STL approach using $\mathrm{BaTiO}_{3} \mathrm{NPs}$ and photoactive dyes is presented. The material's mechanical properties have been observed to follow the Sato and Furukawa model when increasing the NPs loading. Simulation techniques confirmed the experimental acoustic behaviour of the new 3D-printed sensor, which reproduces the behaviour observed on the locust TM, presenting higher amplitudes of motion at low frequencies on the thick region, and the opposite trend on the thin region, leading to acoustic frequency selection. The piezoelectric response of the 3Dprintable material was successfully tested by both direct and converse piezoelectric processes, making it suitable for several applications in a cheaper and more time-efficient process. Further research is required to improve the $d_{33}$ coefficient of the resulting polymer-based composite without losing 3Dprinting resolution. This work also presents relevant information about the biological processes that take place on the locust's TM, revealing that TWs appear not only in active biological systems but also in passive mechanical systems that can be used as sensors and/or actuators, where different regions are able to detect different frequencies at higher amplitudes of motion and therefore leading to acoustic frequency selectivity. Hence, developing a system with multiple regions instead of only two is possible in order to accomplish more accurate frequency discrimination. Thus, we have shown that simple bio-inspired devices can lead to complex systems able to discern between high and low 
acoustic frequencies, giving one more insight into bionic hearing systems.

\section{Acknowledgements}

The authors would like to thank Dr. Milovan Cardona for his guidance and help using the mechanical testing techniques at the University of Strathclyde Biomedical Engineering group.

This research is funded by the European Research Council under the European Union's Seventh Framework Programme (FP/2007-2013) / ERC Grant Agreement n. [615030].

\section{References}

[1]. J. K. Carroll, and A. K. Gaharwar, Macromol. Bioinspired polymeric nanocomposites for regenerative medicine, Chem. Phys., 216 (2015). 248264.

[2]. Q. Ye, F. Zhou, and W. Liu, Bioinspired catecholic chemistry for surface modification, Chem. Soc. Rev., 40 (2011), 4244-4258.

[3]. L. A. Estroff, and A. D. Hamilton, At the interface of organic and inorganic chemistry: Bioinspired synthesis of composite materials, Chem. Mater., 13 (2001), 3227-3235.

[4]. T. Sung, L. Feng, X. Gao, and L. Jiang, Bioinspired surfaces with special wettability, Acc. Chem. Res., 38 (2005), 644-652.

[5]. C. Sanchez, H. Arribat, and M. M. G. Guille, Biomimetism and bioinspiration as tools for the design of innovative materials, Nat. Mater., 4 (2005), 277.

[6]. M. Calisti, M. Giorelli, G. Levy, BMazzolai, B. Hochner, C. Laschi, and P. Dario, Ac octopus-bioinspired solution to movement and manipulation for soft robots, Bioinspir. Biomim., 6 (2011), 036002.

[7]. V. Kopman, and M. Porfiri, Design, modelling, and characterization of a miniature robotic fish for research and education in biomimetics and bioinspiration, IEEE/ASME Transactions on mechatronics, 18 (2013), 471-483.

[8]. G. J. Krijnen, M. Dijkstra, J. J. van Baar, S. S. Shankar, W. J. Kuipers, W. J. de Boer, D. Altpeter, T. S. Lammerink, and R. Wiegerink, MEMS based hair flowsensor as model systems for acoustic perception studies, Nanotechnology, 17 (2006), S84.

[9]. M. Dijkstra, J. J. van Baar, R. Wiegerink, T. S. Lammerink, J. J. de Boer, and G. J. M. Krijnen, Artificial sensory hairs based on the flow sensitive receptor hairs of crickets, J. Micromech. Microeng., 15 (2005), S132.

[10].R. R. Hoy, and D. Robert, Tympanal hearing in insects, Annu. Rev. Entomol., 41 (1996), 433-450.

[11].A. Michelsen, J. Comp. Physiol. A Neuroethol. Sens., Neural. Behav. Physiol., 71 (1971), 63-101.

[12].R. O. Stephen, and H. C. Bennet-Clark, The anatomical and mechanical basis of stimulation and frequency analysis in the locust ear, J. Exp. Biol., 99 (1982), 279314.

[13].J. F. Windmill, M. C. Gopfert, and D. Robert, Tympanal travelling waves in migratory locusts, J. Exp. Biol., 208 (2005), 157-168.

[14].S. V. Murphy, and A. Atala, 3D bioprinting of tissues and organs, Nat. Biotechnol., 32 (2014), 773-785.

[15].S. Bose, S. Vahabzadeh, and A. Bandyopadhyay, Bone tissue engineering using 3D printing, Mater. Today, 16 (2013), 496-504.

[16].M. Guvendiren, J. Molde, R. M. D. Soares, and J. Kohn, Designing biomaterials for 3D printing, ACS Biomater. Sci. Eng., 2 (2016), 1679-1693.

[17].S. C. Ligon-Auer, M. Schwentenwein, C. Gorsche, J. Stampfl, and R. Liska, Toughening of photo-curable polymer networks: a review, Polym. Chem., 7 (2016), 257-286.

[18].Y. S. Rim, S. H. Bae, H. Chen, N. De Marco, and Y. Yang, Recent progress in materials and devices toward printable and flexible sensors, Adv. Mater., 28 (2016), 4415-4440.

[19].J. A. Lewis, and B. Y. Ahn, Device fabrication: Threedimensional printed electronics, Nature, 518 (2015), 42-43.

[20].A. Uriondo, M. Esperon-Miguez, and S. Perinpanayagam, The present and future of additive manufacturing in the aerospace sector: A review of important aspects, Proc. Inst. Eng. Part G, 229 (2015), 2132-2147.

[21].M. H. Elahinia, M. Hashemi, M. Tabesh, and S. B. Bhaduri, Manufacturing and processing of $\mathrm{NiTi}$ implants: a review, Prog. Mater. Sci., 57 (2012), 911946.

[22].I. Roppolo, A. Chiappone, A. Angelini, S. Stassi, F. Frascella, C. F. Pirri, C. Ricciardi, and E. Descrovi, 3D printable light-responsible polymers, Mater. Horiz, 4 (2017), 396.

[23].J. J. Wang, F. Y. Meng, X. Q. Ma, M. X. Xu, and L. Q. Chen, Lattice, elastic, polarization, and electrostrictive properties of $\mathrm{BaTiO} 3$ from first-principles, J. Appl. Phys., 108 (2010), 034107; doi: 10.1063/1.3462441.

[24].Z. Y. Shen, and J. F. Li, Enhancement of piezoelectric constant d33 in BaTiO3 ceramics due to nano-domain structure, J. Ceram. Soc. Jpn., 118 (2010), 940-943.

[25].L. Dong, D. S. Stone, and R. S. Lakes, Enhanced dielectric and piezoelectric properties of $\mathrm{x} \mathrm{BaZrO3-(1-}$ x)BaTiO3 ceramics, J. Appl. Phys., 111 (2012), 084107.

[26].H. Fu, and L. Bellaiche, Ferroelectricity in barium titanate quantum dots and wires, Phy. Rev. Lett., 91 (2003), 257601.

[27].H. T. Martirena, and J. C. Burfoot, Grain-size effects on properties of some ferroelectrics ceramics, J. Phys. C: Solid State Phys., 7 (1974), 3182-3192.

[28].P. Dineva, D. Gross, R. Muller, and T. Rangelov, Dynamic Fracture of Piezoelectric Materials, Springer International Publishing, Switzerland, 1, Chapter 2: Piezoelectric Materials (2014), 7-32.

[29].J. F. Capsal, E. Laffont, L. Dandurand, and C. Lacabanne, Nanotexture influence of $\mathrm{BaTiO} 3$ particles on piezoelectric behaviour of PA 11/BaTiO3 nanocomposites, J. Non-Cryst. Solids, 356 (2010), 629634.

[30].R. Saha, and W. D. Nix, Effects of the substrate on the determination of thin film mechanical properties by nanoindentation, Acta Mater., 50 (2002), 23-38.

[31].W. C. Oliver, G. M. Pharr, An improved technique dor determining hardness and elastic modulus using load and displacement sensing indentation experiments, J. Mat. Res., 7 (1992), 1564-1583.

[32].Y. Sato, and J. Furukawa, A molecular theory of filler reinforcement based upon the conception of internal deformation (a rough approximation of the internal deformation), Rubber Chem. Technol., 36 (1963), 1081-1106.

[33].R. Herdier, D. Jenkins, E. Dogheche, and D. Remiens, Laser Doppler vibrometry for evaluating the piezoelectric coefficient d33 on thin film, Rev. Sci. Instrum., 77 (2006), 093905.

[34].K. M. Rittenmyer, and P. S. Dubbelday, Direct measurement of the temperature-dependent piezoelectric coefficients of composite materials by laser Doppler vibrometry, J. Acoust. Soc. Am., 91 (1992), 2254.

[35].C. Credi, A. Fiorese, M. Tironi, R. Bernasconi, L. Magagnin, M. Levi, and S. Turri, 3D printing of cantilever-type microstructures by stereolithorgaphy of ferromagnetic photopolymers, ACS Appl. Mater. Interfaces, 8(2016), 26332-26342.

[36].Y. Zare, Study of nanoparticles aggregation/agglomeration in polymer particulate 
nanocomposites by mechanical properties, Composites: Part A, 84 (2016), 158-164.

[37].G. A. Manley, R. R. Fay, and A. N. Popper, Active Processes and Otoacoustic Emissions, Springer, New York, 2008. 
Electronic Supporting Information

Bio-inspired 3D-printed piezoelectric device for acoustic frequency selection

Roger Domingo-Roca, ${ }^{a}$ Benjamin Tiller, Joseph Curt Jackson ${ }^{\mathrm{a}}$ and James Frederick Charles Windmilla

a Centre for Ultrasonic Engineering, Dept. Electronic \& Electrical Engineering, 204 George Street,, University of Strathclyde, Glasgow, G1 1XW, United Kingdom. E-mail: roger.domingo-roca@strath.ac.uk 


\section{Morphological study}
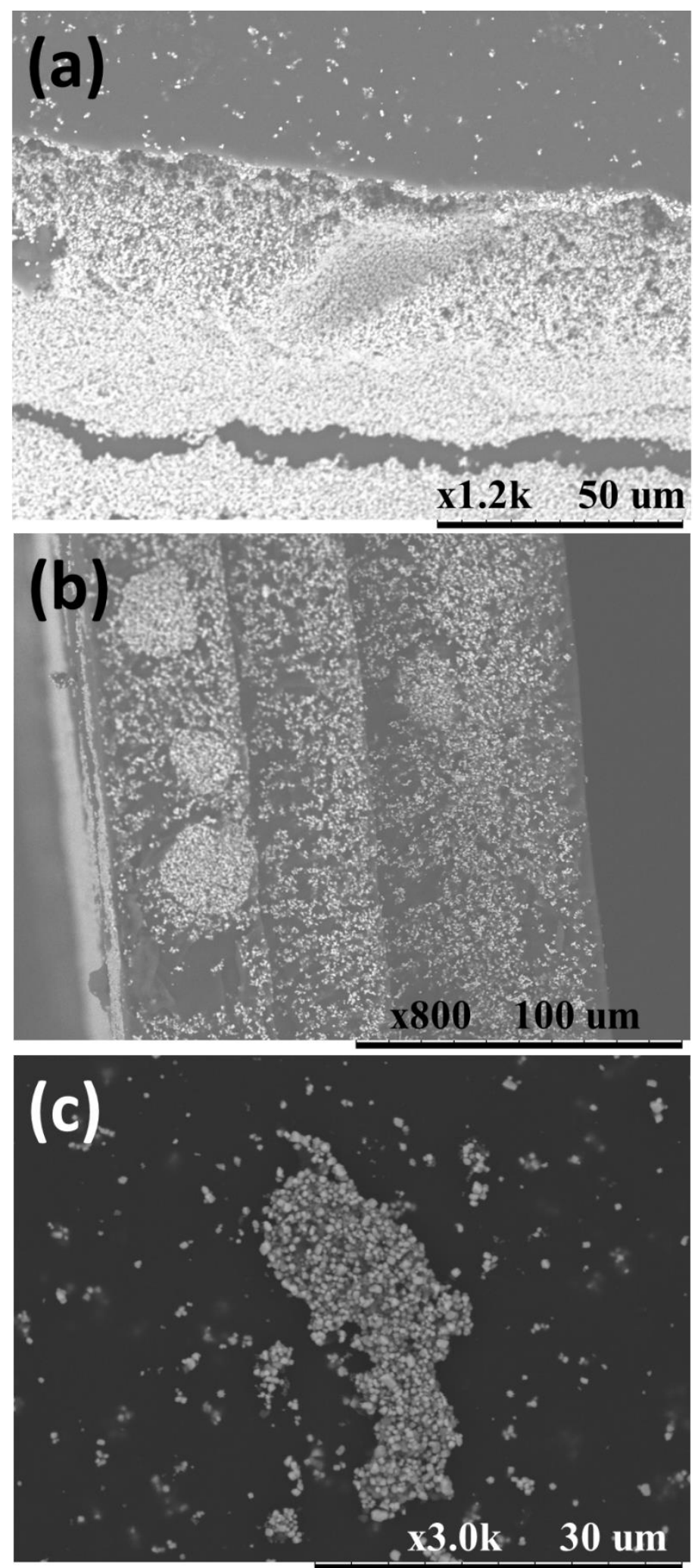

Fig. S1: (a) Shows an SEM on-top image of the control BEMA:BTO sample, where one of the layers has been slightly scratched off using a scalpel. (b) Shows an SEM cross-section image of the control BEMA:BTO sample where the different screenprinted layers can be observed. Because of the rudimentary steps of this process, it can be observed that the layers do not present the exact same layer thickness. (c) Shows a cluster of $\mathrm{BaTiO}_{3} \mathrm{NPs}$. Same image as Fig. $3 \mathrm{~b}$ in the main article. 

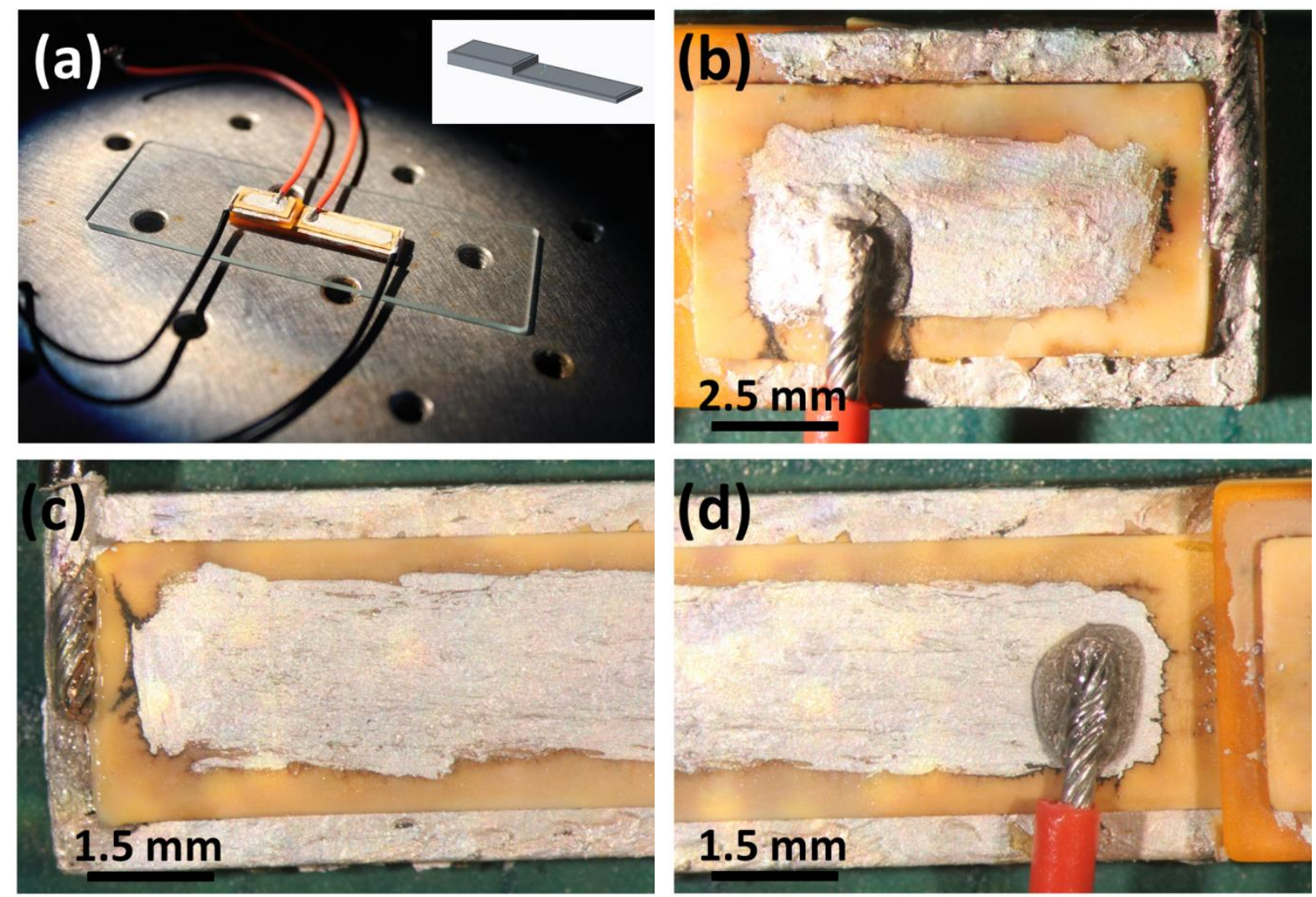

Fig. S2: (a) Isometric view of the 3D-printed hybrid bio-inspired sensor with its corresponding CAD file, as shown in Fig. 1c in the main article. (b) Microscope top view of the thick region of the BEMA:BTO sensor. (c) and (d) show a microscopy top view of the thin region of the BEMA:BTO sensor. In (b), (c), and (d) the silver paint electrodes can be also observed, as well as the wires attached on both the top and the bottom.

Fig. S1a shows the presence of $\mathrm{BaTiO}_{3}$ NPs embedded within the polymeric matrix, whereas Fig. S1b shows the non-uniform distribution of the NPs within the polymer matrix, tending to aggregation (Fig. S1c).

From Fig. S2b,c,d a dark halo can be observed around the silver painted electrodes as a result of the poling process, darker closer to the wires due to an increase of the electric field as a consequence of the edge effects.

The dimensions of the device are shown in Fig. S3. 

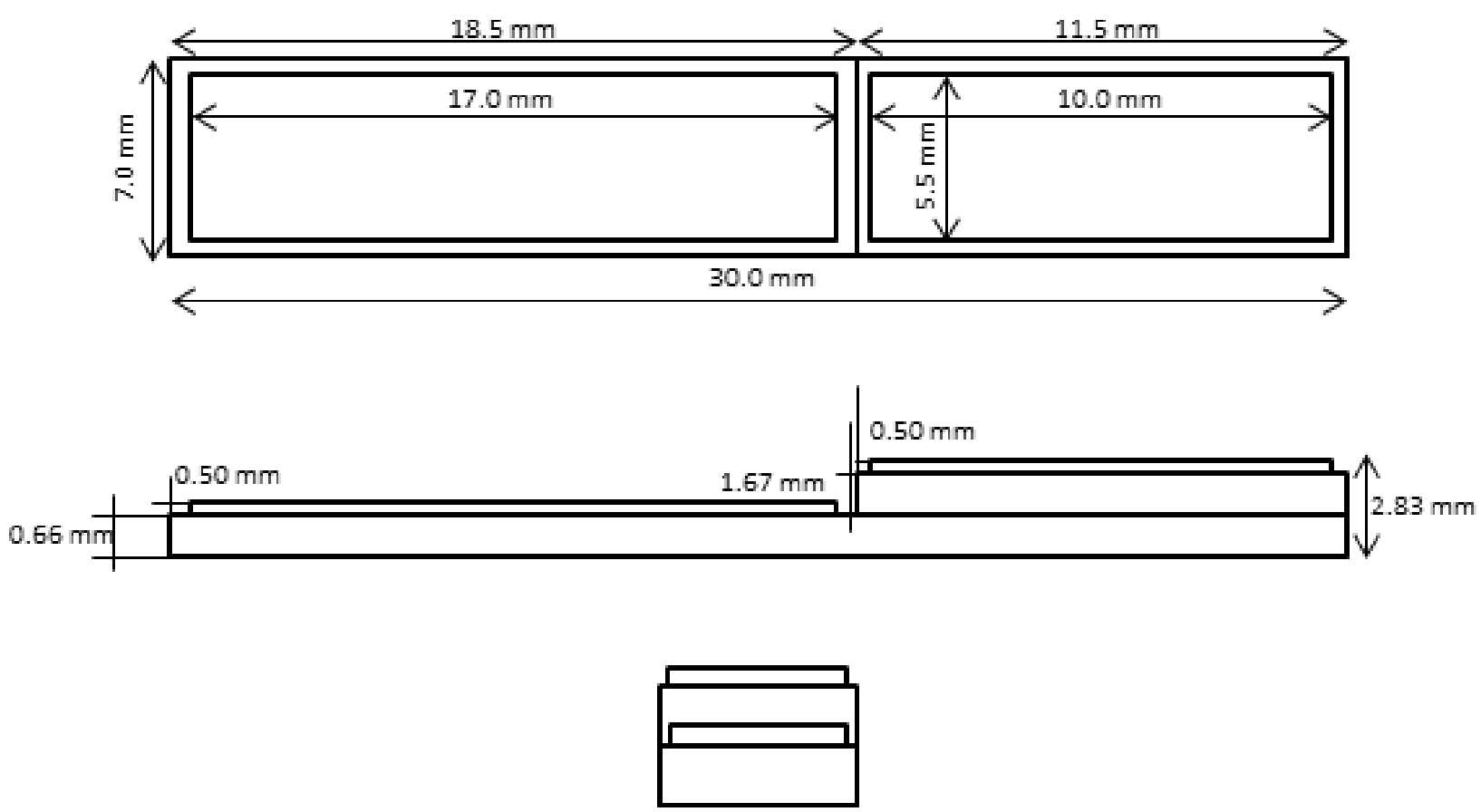

Fig. S3: Shows the dimensions of the 3D-printed samples including all the 3D-printed layers in order to standardize the device. From top to bottom: the top, side, and front views of the 3D-printed samples are shown, respectively.

\section{PVDF data}

(a)

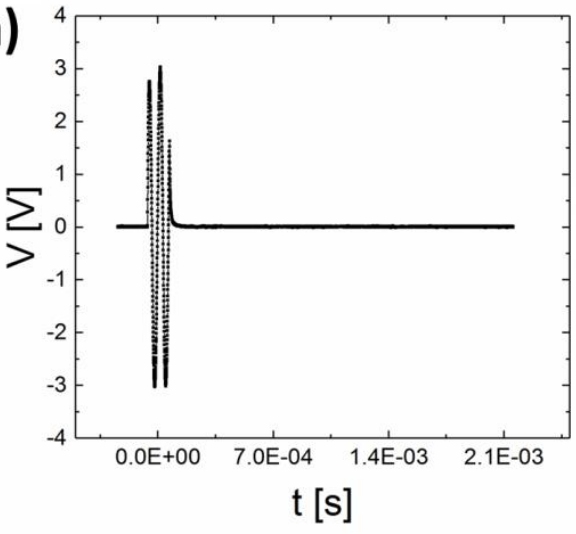

(b)

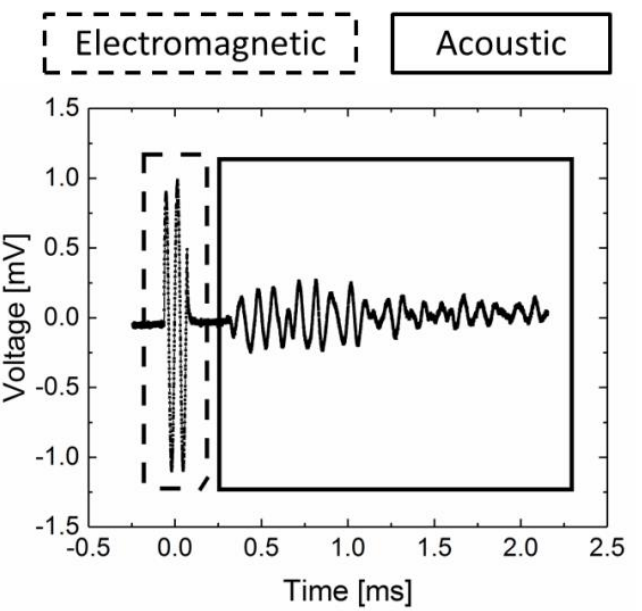

Fig. S4: (a) Shows the acoustic input signal at $15 \mathrm{kHz}$ consisting of a burst of 2 cycles every $100 \mathrm{~ms}$, and (b) shows the response of the device when two PVDF films are attached onto it, which is divided into the electromagnetic (dash-line box) and the acoustic response (solid-line box). The same experiment was repeated for all the sensors in the range of frequencies from 1 $\mathrm{kHz}$ to $17 \mathrm{kHz}$. 


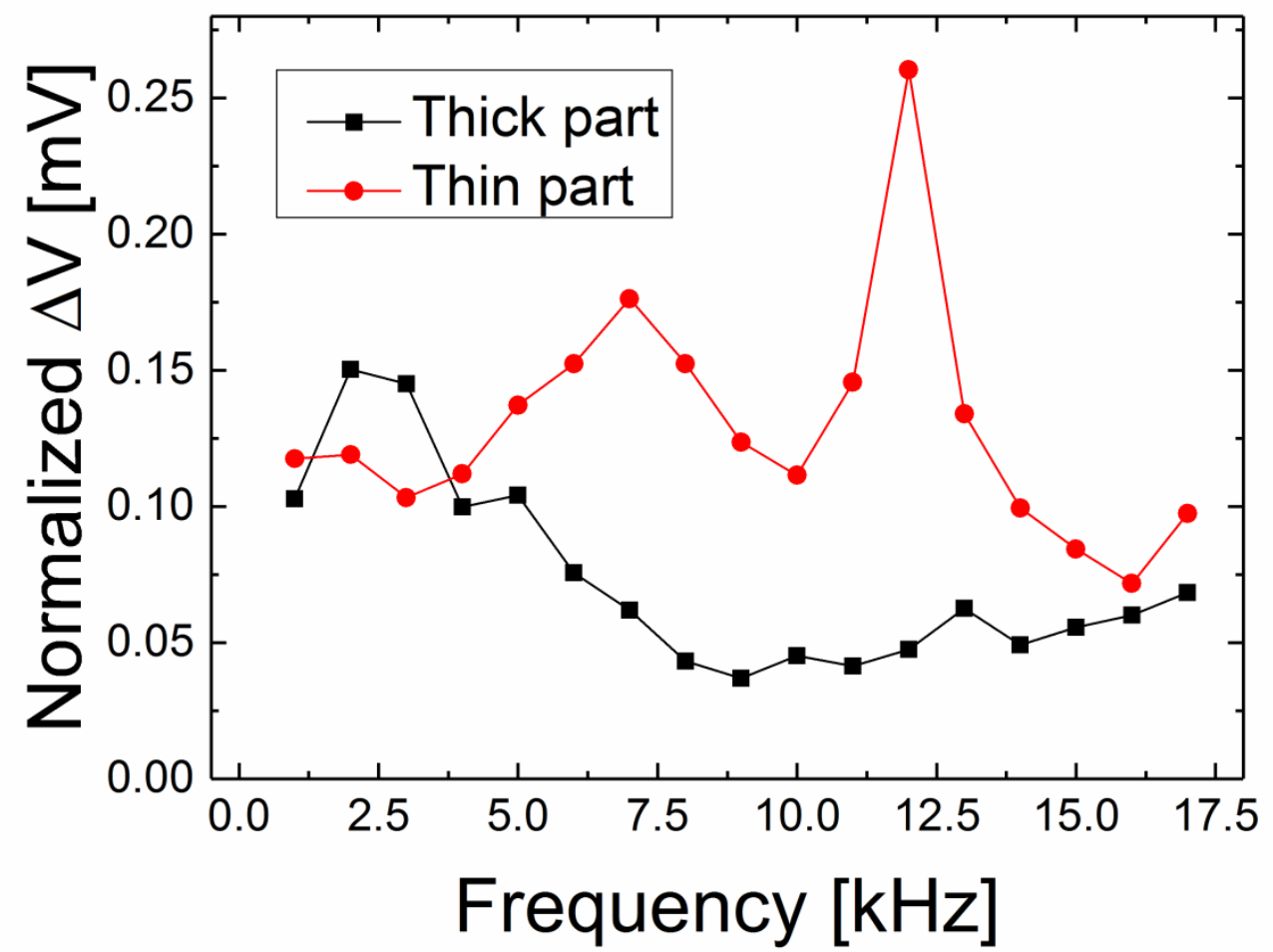

Fig. S5: Normalized values of the output voltage of the different regions of the sensor with PVDF films under acoustic stimulation within the frequency range of $1 \mathrm{kHz}$ to $17 \mathrm{kHz}$ in steps of $1 \mathrm{kHz}$ revealing larger voltage outputs at lower frequencies in the thick region and vice versa.

Fig. S5 shows the normalized values of the output voltage when the sensor was stimulated under an acoustic field, leading to frequency selectivity, as the thick membrane shows larger amplitudes of motion at low frequencies, and the thin membrane shows larger amplitudes of motion at high frequencies, showing a sub-peak at $7 \mathrm{kHz}$. 